\title{
Does a hadron-quark phase transition in dense matter preclude the existence of massive neutron stars?
}

\author{
N. Chamel ${ }^{1}$, A. F. Fantina ${ }^{1}$, J. M. Pearson ${ }^{2}$ and S. Goriely ${ }^{1}$ \\ ${ }^{1}$ Institut d'Astronomie et d'Astrophysique, CP-226, Université Libre de Bruxelles, \\ 1050 Brussels, Belgium \\ ${ }^{2}$ Dépt. de Physique, Université de Montréal, Montréal (Québec), H3C 3J7 Canada
}

\begin{abstract}
We study the impact of a hadron-quark phase transition on the maximum neutronstar mass. The hadronic part of the equation of state relies on the most up-to-date Skyrme nuclear energy density functionals, fitted to essentially all experimental nuclear mass data and constrained to reproduce the properties of infinite nuclear matter as obtained from microscopic calculations using realistic forces. We show that the softening of the dense matter equation of state due to the phase transition is not necessarily incompatible with the existence of massive neutron stars like PSR J1614-2230.
\end{abstract}

Keywords. stars: neutron, dense matter, equation of state, stars: interiors, gravitation

\section{Introduction}

Neutron stars (NSs) result from the gravitational collapse of massive stars with $M \gtrsim$ $8 M_{\odot}$ at the end point of their evolution. They are among the most compact objects in the universe, with a central density which can reach several times the nuclear saturation density. At least, three different regions can be identified in the interior of a neutron star: (i) the "outer crust", at densities above $\sim 10^{4} \mathrm{~g} \mathrm{~cm}^{-3}$, composed of fully ionized atoms, arranged in a Coulomb lattice of nuclei, neutralized by a degenerate electron gas, (ii) the "inner crust", at above $\sim 4 \times 10^{11} \mathrm{~g} \mathrm{~cm}^{-3}$, composed of neutron-proton clusters and unbound neutrons, neutralized by a degenerate electron gas, and (iii) the core, at densities above $\sim 10^{14} \mathrm{~g} \mathrm{~cm}^{-3}$. The precise measurement of the mass of pulsar PSR J1614-2230 by Demorest et al. (2010) has revived the question of the composition of the core. Just below the crust, the matter consists of a mixture of neutrons, protons, electrons and possibly muons. The composition of the central region of a NS is still a matter of debate (see e.g. Haensel et al. 2007).

In the present work, we study the impact of a hadron-quark phase transition in dense matter on the maximum mass of cold isolated NSs (see Chamel et al. 2012 for a general discussion of the maximum mass of hybrid stars).

\section{Hadronic equation of state}

The global structure of a NS is determined by the equation of state (EoS), i.e. the relation between the matter pressure $P$ and the mass-energy density $\rho$. Before considering the possibility of a phase transition from hadronic to quark matter in the core of NSs, we will begin with the hadronic EoSs. A good starting point is the family of three EoSs, BSk19, BSk20 and BSk21, which have been developed to provide a unified treatment of all regions of a NS (see Pearson et al. 2011, Pearson et al. 2012). These EoSs are based 
on nuclear energy-density functionals derived from generalized Skyrme forces (in that they contain additional momentum- and density-dependent terms), which fit essentially all measured masses of atomic nuclei with an rms deviation of $0.58 \mathrm{MeV}$ for all three models. Moreover, these functionals were constrained to reproduce three different neutron matter EoSs, as obtained from microscopic calculations (see Goriely et al. 2010). All three EoSs assume that the core of a NS is made of nucleons and leptons. The BSk19 EoS was found to be too soft to support NSs as massive as PSR J1614-2230 (Chamel et al. 2011) and therefore, it will not be considered here.

\section{Hadron-quark phase transition}

Given the uncertainties in the composition of dense matter in NSs, we will simply suppose that above the average baryon density $n_{\mathrm{N}}$, matter undergoes a first-order phase transition to deconfined quark matter subject to the following restrictions: (i) for the transition to occur the energy density of the quark phase must be lower than that of the hadronic phase, (ii) according to perturbative quantum chromodynamics (QCD) calculations (e.g. Kurkela et al. 2010), the speed of sound in quark matter cannot exceed $c / \sqrt{3}$ where $c$ is the speed of light. At densities below $n_{\mathrm{N}}$, matter is purely hadronic while a pure quark phase is found at densities above some density $n_{\mathrm{X}}$. In the intermediate region $\left(n_{\mathrm{N}}<n<n_{\mathrm{X}}\right)$ where the two phases can coexist, the pressure and the chemical potential of the two phases are equal: $P_{\text {quark }}(n)=P_{\text {hadron }}\left(n_{N}\right)$ and $\mu_{\text {quark }}(n)=\mu_{\text {hadron }}\left(n_{N}\right)$. The EoS of the quark phase at $n>n_{\mathrm{X}}$ is given by:

$$
P_{\text {quark }}(n)=\frac{1}{3}\left(\mathcal{E}_{\text {quark }}(n)-\mathcal{E}_{\text {quark }}\left(n_{\mathrm{X}}\right)\right)+P_{\text {hadron }}\left(n_{\mathrm{N}}\right) .
$$

We set the density $n_{\mathrm{N}}$ to lie above the highest density found in nuclei as predicted by Hartree-Fock-Bogoliubov calculations, namely $n_{\mathrm{N}}=0.2 \mathrm{fm}^{-3}$ (BRUSLIB). The density $n_{\mathrm{X}}$ is adjusted to optimize the maximum mass under the conditions mentioned above. Eq. (3.1) turns out to be very similar to that obtained within the simple MIT bag model, which has been widely applied to describe quark matter in compact stars (see e.g. Haensel et al. 2007). The effective bag constant $B$ associated with the BSk21 hadronic EoS is $56.7 \mathrm{MeV} \mathrm{fm}^{-3}$.

\section{Maximum mass}

Considering the stiffest hadronic EoS (BSk21), we have solved the TolmanOppenheimer-Volkoff equations (Tolman 1939, Oppenheimer \& Volkoff 1939) in order to determine the global structure of a non-rotating neutron star. The effect of rotation on the maximum mass was found to be very small for stars with spin-periods comparable to that of PSR J1614-2230 (Chamel et al. 2011); we therefore neglect it. The gravitational mass versus circumferential radius relation is shown in Fig. 1. We have considered two cases: a purely hadronic neutron star described by our BSk21 EoS (dashed line) and a hybrid star with a quark core (solid line). The corresponding maximum masses are $2.28 M_{\odot}$ and $2.02 M_{\odot}$ respectively. In both cases, the existence of two-solar mass NSs is therefore allowed.

\section{Conclusions}

The presence of a deconfined quark-matter phase in NS cores leads to a maximum mass of about $2 M_{\odot}$, which is still compatible with the mass measurement of PSR J1614-2230 


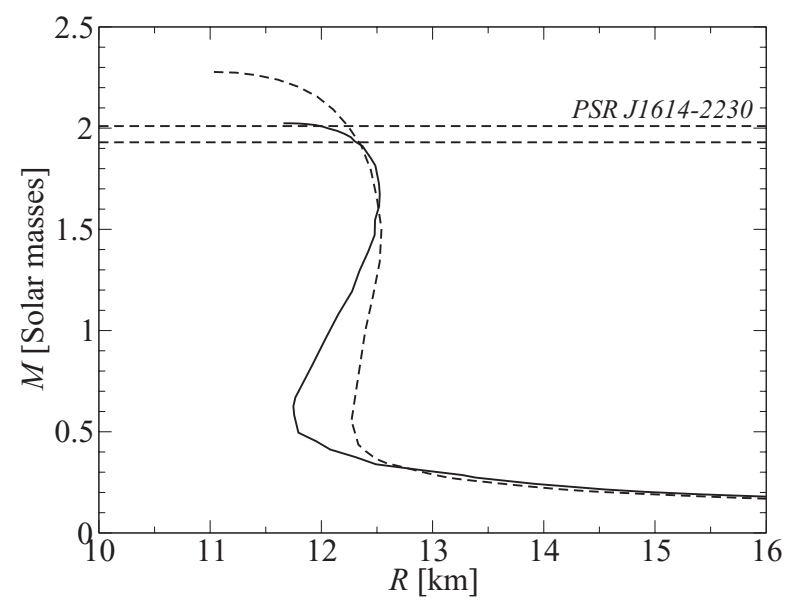

Figure 1. Gravitational mass versus circumferential radius with (solid line) and without

(dashed line) a quark-matter core. See the text for detail.

by Demorest et al. (2010), but which could be challenged by observations of significantly more massive NSs (see Clark et al. 2002, Freire et al. 2008, van Kerkwijk et al. 2011) unless the sound speed in quark matter is significantly larger than that predicted by perturbative QCD calculations (Kurkela et al. 2010).

\section{Acknowledgements}

FNRS (Belgium), NSERC (Canada) and CompStar, a Research Networking Programme of the European Science Foundation are gratefully acknowledged.

\section{References}

BRUSLIB http://www.astro.ulb.ac.be/bruslib

Chamel, N., Fantina, A. F., Pearson, J. M., \& Goriely, S. 2011, Phys. Rev. C, 84, 062802

Chamel, N., Fantina, A. F., Pearson, J. M., \& Goriely, S. 2012, arXiv:1205.0983

Clark, J. S., Goodwin, S. P., Crowther, P. A., Kaper, L, Fairbairn, M., Langer, N., \& Brocksopp, C. 2002, Astron. Astrophys., 392, 909

Demorest, P. B., Pennucci, T., Ransom, S. M., Roberts, M. S. E., \& Hessels, J. W. T. 2010, Nature, 467, 1081

Freire, P. C. C., Ransom, S. M., Bégin, S., Stairs, I. H., Hessels, J. W. T., Frey, L. H., \& Camilo, F. 2008, Astrophys. J., 675, 670

Goriely, S., Chamel, N., \& Pearson, J. M. 2010, Phys. Rev. C, 82, 035804

Haensel, P., Potekhin, A. Y., \& Yakovlev, D. G. 2007, Astrophys. Space Sc. L., 326

Kurkela, A., Romatschke, P., \& Vuorinen, A. 2010, Phys. Rev. D, 81, 105021

Oppenheimer, J. R. \& Volkoff, G. M. 1939, Phys. Rev., 55, 374

Pearson, J. M., Goriely, S., \& Chamel, N. 2011, Phys. Rev. C, 83, 065810

Pearson, J. M., Chamel, N., Goriely, S., \& Ducoin, C. 2012, Phys. Rev. C, 85, 065803

Tolman, R. C. 1939, Phys. Rev., 55, 364

van Kerkwijk, M. H., Breton, R. P., \& Kulkarni, S. R. 2011, Astrophys. J. 728, 95 